University of Nebraska - Lincoln

DigitalCommons@University of Nebraska - Lincoln

Papers in Biomaterials

Chemical and Biomolecular Engineering

Research and Publications

March 1999

\title{
Liquid-Phase Catalytic Oxidation of Unsaturated Fatty Acids
}

Hossein Noureddini

Department of Chemical Engineering, University of Nebraska-Lincoln, hnouredd@unlnotes.unl.edu

M Kanabur

Department of Chemical Engineering, University of Nebraska-Lincoln

Follow this and additional works at: https://digitalcommons.unl.edu/chemeng_biomaterials

Part of the Biomaterials Commons

Noureddini, Hossein and Kanabur, M, "Liquid-Phase Catalytic Oxidation of Unsaturated Fatty Acids " (1999). Papers in Biomaterials. 8.

https://digitalcommons.unl.edu/chemeng_biomaterials/8

This Article is brought to you for free and open access by the Chemical and Biomolecular Engineering Research and Publications at DigitalCommons@University of Nebraska - Lincoln. It has been accepted for inclusion in Papers in Biomaterials by an authorized administrator of DigitalCommons@University of Nebraska - Lincoln. 


\title{
Liquid-Phase Catalytic Oxidation of Unsaturated Fatty Acids
}

\author{
H. Noureddini* and M. Kanabur \\ Department of Chemical Engineering University oi Nebrastra-Lincoln, Lincoin, Nebraska $68588-0126$
}

\begin{abstract}
Liquid-phase catalytic oxidation of oleic acid with hydrogen peroxide in the presence of various transition metal/metal oxide catalysts was studied in a batch autoclave reactor. Azelaic and pelargonic acids are the major reaction products. Tungsten and tantalum and their oxides in supported and unsupported forms were used as catalysts. Alumina pe!lets and Kieseiguhr powder were used as supports for the catalysts. Tungsten, tantaium, molybdenum, zirconium, and niobium were also examined as catalysts. Tertiary butanol was used as solvent. Experimental results concluded that tungsten and tungstic oxide are more suitable catalysts in terms of their activity and selectivity. The rate of reaction observed in the case of supported catalysts appears to be comparable or superior to that of unsuoported catalysts. In pure form, tungsten, tantalum, and moiybdenum showed strong catalytic activity in the oxidation reaction; however, except for tantalum the other two were derermined to be economically unfeasible. Zirconium and niobium showed very littie catalytic activity. Based on the experimental observation, tungstic oxide supported on silica is the rrost suitable catalyst for the oxidation of oleic acid with $85 \%$ of the starting oleic acid converted to the oxidation products in 60 min of reaction with high selectivity ior azeiaic acid.

Paper no. 18385 in JAOCS 76, 305-312 (March 1999).
\end{abstract}

KEY WORDS: Azelaic acid, catalvtic oxidation, oleic acid, pelargonic acid, silica-5upported cataly'st, tungsten oxide, unsalurated ratty acids.

With the expected depletion of petroleum reservoirs in the next few decades and growing concern for the associated environmental poliution, much emphasis has been placed on the utilization of renewable resources such as agricultural mater. ial as an alternative to the planetary resources. In this context, vegetable oils show a lol of promise as an alternative feed. stock. Efforts are underway to utilize the abundantly available vegetable oils and oil-based producis for the production of mono-and dibasic acids that in turn find majer potential application as betrer substitutes for the petroleum-based feedstock.

Oleic and erucic acids are the major monounsaturated fatty acid components of soybean and crambe oils. respectively. Unsaturated fatty acids may undergo various reactions with

*Ta whom correspordence should be addressed.

E-mail: hnoureddini (2) unl.edu several compounds forming a wide range of products. These reactions may involve either the carboxylic group or the cabon-carbon double bond in an unsaturated fatty acid. Reactiuns such as hydrogenation, epoxidation, or oxidation may occur at the carbon-carbon double bond. The oxidation of unsaturated fatty acids results in partial or complete cleavage of the carbon-carbon double bond. Mono- and dicarboxylic acids are the main products of the complete oxidation of the unsaturated fatty acids.

Dicarboxylic acids and their derivatives have many industrial applications (i-3). Diesters and Jinear polyesters of straight-chain fatty acids are used as plasticizers for polyvinyl chloride (3-5). Esters of dicarboxylic acids. with a specific amount of branching. are used as lubricants and hydraulic fiuids over a wide temperature range $(3,6)$. Applications of dicarboxylic acids are as polymer intermediates in polyamides. polyesters, polyurethane coatings, and alkyd resins. For exampie. nylon 1313 produced from brassylic acid. shows enhanced properties sompared to those of commoniy available nvlons, e.g. a lower melting point, lower densily. and more hydrophubicity than nylon-11 and nylon-12 (7), properties advantageous in specific applications. Further, the reaction involved in the synthesis of nylon-1313 is much simpler compared to the reactions associated with nylon- 11 and -12 .

The conventional process for the production of dicarboxylic acid from unsaturated fatty acids involves oxidative ozonolysis of unsaturated fatty acids. a process used for manufacturing azelaic acid from oleic acid (8). Nieschlag and coworkers (9) utilized erucic acid in a continuous pilot-plant scale ozonolysis process for brassylic acid production. The use of ozone as oxidant provides some advantages, namely. high reactivity. selectivily, and lack of by-products. However. owing to the inherent health and safety hazards. high capitial cosis, handling of large volumes of reaction gases and high energy requirements associated with the process, effort.s are being made to find a safer and economically more viable process for the production of diacids.

Oxidation of usscaturated facty acids with a variety of oxidizing agents such as potassium permanganate, polassium dichromate. chromic acid, and sodium hypochlorite appears in the licerature. Garti and Avni (10) investigated the oxidation of oleic acid with potassium permanganate. The oxidation of oleic acid in neutral orl in water emulsion resulted in 
the formation of dihydroxy, ketohydroxy, and diketo acids along with azelaic and pelargonic acids. Zaldman et al. (11) used sodium hypochlorite as an oxidizing agent for the oxidation of oleic acid. The results showed that oxidation of oleic acid in an emulsion system consisting of sodium hypochlorite, surface-active agents, and ruthenium chloride as catalysts could be achieved with higher conversion and reduced reaction time compared with the nonemulsified (heterogeneous) systems. The reaction of alkaline potassium permanganate with ricinoleic acid was investigated by Hill and McEwen (12), resulting in about $34 \%$ yield of azelaic acid. Ayorinde et al. (13) synthesized azelaic and suberic acids from oxidation of vernonia oil using nitric acid as oxidant. The isolated yields of azelaic and suberic acids were about 15 and $11 \%$, respectively. The reaction by-products consisted of many short- and long-chain mono- and dicarboxylic acids. Further, Passi et al. (14) carried out studies by subjecting free or esterified polyunsaturated fatty acids to the oxidative attack by hydroxyl radicals generated by Fenton reaction, radiation-induced oxidation, and enzymatic oxidation. They demonstrated that, upon lipid peroxidation. polyunsaturated fatty acids originate specific dicarboxylic acids that may be regarded as a distinctive feature of the particular positions of the double bonds in the molecules. A hypothetical scheme for the formation of dicarboxylic acids as a result of the abovementioned reactions was presented.

Liquid-phase oxidation of hydrocarbons to dibasic acids using heterogenized catalysts has received a lot of attention in recent years. Catalysts such as transition metal oxides, cation-exchanged zeolites, and transition-metal complexes with cross-linked polymer are suitable for the liquid-phase hydrocarbon oxidation. Advantages such as high selectivity associated with homogeneous catalysts and ease of recovery and regeneration in the case of heterogeneous catalysts can be combined in the case of heterogenized homogeneous catalysts. Shen and Weng $(15,16)$, reported liquid-phase oxidation of cyclohexane and cyclohexanone with supported cobalt (15) and cerium (16) catalysts. Reaction mechanism, rate and conditions, and product distribution are discussed.

Catalytic oxidation of olefins with hydrogen peroxide in the presence of tungstic acid, molybdic acid, and their heteropoly-acids is discussed in a patent by Fujitani and Nakazawa (17). Nakano and Foglia (18) studied the oxidation of unsaturated and hydroxy fatty acids by ruthenium tetroxide and ruthenium oxyanions. At high molar ratio of oxidant to double bond $(4: 1)$, both catalysts quantitatively cleaved the double bonds to give nonanoic and azelaic acid. Rushgen and co-workers (19) studied the liquid-phase oxidative cleavage of unsaturated fatty acids with hydrogen peroxide or peracetic acid and transition-metal catalysts. The oxidation of oleic acid with hydrogen peroxide and tungsten acid resulted in nonanoic and azelaic aids as the main oxidation products.

Liquid-phase oxidation reactions generally proceed with a free-radical mechanism (20). The reactions are generally characterized by an induction period followed by a rapid reaction phase. During the induction period, initiation reactions gradually increase the free-radical concentration until propagation becomes a dominant process. Sometimes the poisons, which get thoroughly adsorbed, can also cause the induction period, and are sufficienctly reactive enough to form less thoroughly adsorbed products. The free-radical mechanisms can be ascertained by inhibitions of such processes by unsaturated monomers or other acceptors of free radicals or initiation of such processes by azo compounds. Willms and coworkers (20) carried out the rate enhancement studies on $m$-xylene oxidation in the presence of hydrogen peroxide and phenol and found that the hydrogen peroxide decomposes instantaneously under the operating conditions of $127-200^{\circ} \mathrm{C}$ and 13 bar pressure to produce the free radicals necessary to initiate the $m$-xylene oxidation, thereby almost eliminating the induction period normally associated with the oxidation process of $m$-xylene.

Liquid-phase oxidation of oleic acid with hydrogen peroxide in the presence of various transition metal/metal oxide catalysts is discussed in this paper. The stoichiometric equation for this reaction is as follows:

$$
\begin{aligned}
& \mathrm{H}_{3} \mathrm{C}\left(\mathrm{CH}_{2}\right)_{7} \mathrm{CH}=\mathrm{CH}\left(\mathrm{CH}_{2}\right)_{7} \mathrm{COOH}+4 \mathrm{H}_{2} \mathrm{O}_{2} \underset{\text { Coulysst }}{\longrightarrow} \\
& \quad \mathrm{H}_{3} \mathrm{C}\left(\mathrm{CH}_{2}\right)_{7} \mathrm{COOH}+\mathrm{HOOC}\left(\mathrm{CH}_{2}\right)_{7} \mathrm{COOH}+4 \mathrm{H}_{2} \mathrm{O}
\end{aligned}
$$

One of the objectives of this study was to investigate the parameters involved in the process scale-up; therefore, catalysts in supported and unsupported forms were both examined. Tungstic oxide in powder form, supported on Kieselguhr (silica) powder and supported on alumina pellets, was tested. Similarly, a comparison study was made between tantalum oxide in powder form and in supported form on alumina. Tungsten, tantalum, molybdenum, zirconium, and niobium were also examined as catalysts. The reaction temperature was $130^{\circ} \mathrm{C}$. and the hydrogen peroxide concentration was at $100 \%$ excess. These conditions were optimized in a previous work (21).

\section{EXPERIMENTAL PROCEDURES}

Materials. Oleic acid (99\%) and boron trifluoride-methanol (14\%) were obtained from Sigma Chemical Company (St. Louis, MO). Oleic acid (90\%) and tungsten oxide powder (99.99\%) were obtained from Aldrich Chemical Company (Milwaukee, WI). Hydrogen peroxide (30\%) and tertiary butanol $(100 \%)$ were purchased from J.T. Baker (Phillipsburg. NJ). The supports for the catalysts, viz. aluminum oxide (alumina) pellets in activated form of $3.2 \mathrm{~mm} .90 \mathrm{~m}^{2}$ per g surface area, $135 \AA$ average pore diameter, Kieselguhr (silica) powder of -325 mesh, $30 \mathrm{~m}^{2}$ per g surface area, $250 \AA$ average pore diameter, tantalum oxide powder $(99.99 \%)$ of puratronic grade, tantalum ethoxide $(99.99 \%)$, and tantalum wire $(99.9 \%$ ) of $0.25 \mathrm{~mm}$ diameter, were obtained from Alfa Aesar (Ward Hill, MA). Tungsten, tantalum, molybdenum, zirconium, and niobium were obtained from Alfa Aesar. The ammonium metatungstate $(99 \%)$ was obtained from Sirem Chemicals (Newburyport, MA). 
The standards for heptanoic methyl ester; hexanoic, octanoic, pelargoric, succinic, glutaric, pimelic, suberic, azelaic. stearic, oleic, and behenic acids were obtained from Sigma Chemical Company, and standards for brassylic and undecane diacids were purchased from Aldrich Chemical Company.

Equipment. A bench-top mini reactor (model number 4562; Part Instrument Company. Moline. IL) was used for the oxidation reactions. The reactor assembly was constructed from type 316 stainless steel with a $450-\mathrm{mL}$ bomb. The reactor was equipped with a magnetic stirter, a four-blade, downward-thrust impeller, and a 1/12-hp variable speed motor with a pulley arrangement to turn the stirrer at speeds from 0 to $800 \mathrm{rpm}$. Heating mantel and internal cooling loop provided the heating and cooling requirements. A Parr 4843 PID controller was used for controlling and monitoring the reaction temperature and the impeller speed. Desired temperatures were programmed into the PID controller, which enabled it to control temperatures as needed. A solenoid valve controlled the flow of cooling water into the reactor. The temperature of the bomb could be monitored to within $\pm 1^{\circ} \mathrm{C}$ accuracy. A nitrogen cylinder provided the purge gas for the process. The purge gas is introduced into the bottom of the reactor bomb. All the preweighed reactants were manually charged into the reactor.

A " $U$ " tube glass apparatus was used for the calcination of supported catalyst. A radiant heater purchased from Omega Engineering Inc. (Stamford. CT) was used for heating the calcination tube. The rate of heating during calcination was controlled by a temperature controller supplied by Watlow (St. Louis. MO). The air flow rate required for calcination was regulated with the help of a rotameter.

Catalyst preparation. Different types of unsupported and supported catalysts were used in this study. Unsupported catalysts used were tantalum, tungsten. molybdenum, zirconium, and niobium in the form of metal wire, and tungstic and tantalum oxides. Supported catalysts used were tantalum oxide on alumina and tungstic oxide on alumina and silica support.

Supported tantalum oxide catalyst was prepared by impregnation of dried alumina pellets with tantalum ethoxide precursor dissolved in ethanol. Each batch of catalyst consisted of about $6 \mathrm{~g}$ of support and $3 \mathrm{~g}$ of tantalum ethoxide. The impregnation was carried out in a beaker. Then impregnated alumina pellets were dried on a hotplate. The dried material was subjected to calcination at $450^{\circ} \mathrm{C}$ for about $3 \mathrm{~h}$ in the calcination tube. The temperature of the calcination chamber was increased at the rate of $5^{\circ} \mathrm{C} / \mathrm{min}$. The airflow rate was maintained at about $7-8 \mathrm{~mL} / \mathrm{min}$ through the calcination process.

Impregnation of tungstic oxide on alumina was similar to the above procedure except that $2 \mathrm{~g}$ of ammonium metatungstate precursor was used in each batch, and distilled water was the solvent. The impregnated support was then dried and calcined. Calcination temperature was $400^{\circ} \mathrm{C}$ for about $2 \mathrm{~h}$. A similar procedure was used to impregnate tungstic oxide on silica support.
Procedures. Experiments were conducted under a batchwise constant temperature and constant pressure mode. The reaction feed consisted of $20 \mathrm{~g}$ of oleic acid. $60 \mathrm{~mL}$ of hydrogen peroxide of $30 \%$ concentration, and $150 \mathrm{~mL}$ of certiary butanol. The reactor was purged with nitrogen prior to heating. Timing was started as soon as the heating procedure began. Most experiments were carried out for $5 \mathrm{~h}$. In the case of supported catalysts, one batch of prepared catalysts, as described in the previous secrion, was charged along with the feed mixture. In the case of experiments with unsupported catalysts, about $1.5 \mathrm{~g}$ of tantalum oxide, $1.5 \mathrm{~g}$ of tungstic oxide. or $1.2 \mathrm{~g}$ of pure metal in the form of a wire was used in the experiments. The reactor contents were agitated continuously with a stirter operating at about $500 \mathrm{rpm}$. Samples were taken periodically from the reaction mixture for analysis.

Sample preparation. Samples from oleic acid oxidation were analyzed by gas chromatography and were expected to contain a variety of mono- and dicarboxylic acids. All samples were converted to their respective methyl esters before analysis with Metcalfe and co-workers' $(22,23)$ derivatization procedures. A $14 \%$ boron trifluoride solution in methanol was used to derivatize fatty acids. To each sample, weighing about $500 \mathrm{mg} .4 \mathrm{~mL}$ of boron trifluoride-methanol solution was added. The mixture was heated to about $80^{\circ} \mathrm{C}$ for about 15 min. Subsequently, the mixture was allowed to cool at room conditions for about $20 \mathrm{~min}$. The esterified product was dissolved in $2 \mathrm{~mL}$ of petroleum ether. Then the sample was extracted two times with $4 \mathrm{~mL}$ of distilled water. The aqueous phase was discarded, and the organic phase containing mainly esters of product acids was dehydrated by adding a small quantity of anhydrous sodium sulfate. Solvent was then removed by passing a steady stream of dry air over the sample container. Fina!ly, the product was dissolyed in exactly I $\mathrm{mL}$ of anhydrous ether solvent and kept under $0^{\circ} \mathrm{C}$ prior to its analysis.

Analisis. Gas chromatography with flame-ionization detectors has been widely used for the separation and quantification of methyl esters of fatty acids $(24,25)$. A HewlettPackard HP 6890 series GC system equipped with HP INNOWax capillary column $(30 \mathrm{~m} \times 0.25 \mathrm{~mm}$ i.d. $\times 0.25 \mathrm{~mm})$. HP Series II GC Electronic Pressure Control (EPC) and splitsplitless inlet (Palo Alto, CA) was used for the analysis of products. Helium was used as a carrier gas with a velocity of $42 \mathrm{~cm} / \mathrm{s}$ and a pressure of 24 psi with EPC at constant flow mode $(1.9 \mathrm{~mL} / \mathrm{min})$. A split ratio of $20: 1$ was maintained. The hydrogen flow and air flow rates were kept at 30 and 275 $\mathrm{mL} / \mathrm{min}$, respectively. The front inlet temperature was maintained at $220^{\circ} \mathrm{C}$. The oven temperature was maintained at $50^{\circ} \mathrm{C}$ for $1 \mathrm{~min}$, prior to injection and after injection. Then a ramp rate of $10^{\circ} \mathrm{C} / \mathrm{min}$ was used until oven temperature reached $160^{\circ} \mathrm{C}$. After a hold-up time of $1 \mathrm{~min}$ at $160^{\circ} \mathrm{C}$, the oven temperature was further increased at the rate of $5^{\circ} \mathrm{C} / \mathrm{min}$ to a final temperature of $260^{\circ} \mathrm{C}$. The duration of sample run was $35 \mathrm{~min}$. An exact amount of $1 \mu \mathrm{L}$ of sample was used each time. The detector temperature was maintained at $280^{\circ} \mathrm{C}$. The quantification of the acids in the samples was car- 
ried out by the HP Chemstation software, which was provided with the calibration runs. Behenic acid was the intemal standard for the analysis.

\section{RESULTS AND DISCUSSION}

Catalytic oxidation of an unsaturated fatty acid with hydrogen peroxide and transition-metal catalyst results in the formation of mono- and dicarboxylic acids (17-19). A typical profile of various products for the oxidation of oleic acid with unsupported tungstic oxide catalyst is presented in Figure 1. This profile summarizes the reaction kinetics after the first hour of the reaction. As this figure indicates, azelaic and nonanoic acids are the main reaction products. Numerous shorter-chain mono- and dicarboxylic acids of $\mathrm{C}_{5}$ to $\mathrm{C}_{8}$ length are also formed during the course of oxidation, indicating that chain degradation also takes place during the course of oxidation. The reaction results for other cases studjed are summarized in Figures 2-6. The formation of the chain degradation products consistently appeared in all cases under investigation. For the purpose of simplicity, only nonanoic, azelaic. octanoic, suberic, and oleic acids are shown in Figures 2-6. As these figures indicate, the unsaturation bond in oleic acid is effectively cleaved. yielding mainly the corresponding mono- and dicarboxylic acids.

Reactivity. In general, during the course of reaction, the concentration of azelaic and nonanoic acids increased to a maximum and then started to decrease. The exact time during which maximal concentration of azelaic and nonanoic acids was reached depended on the type of catalyst used. In the case of tungstic oxide supported on silica, maximal azelaic acid

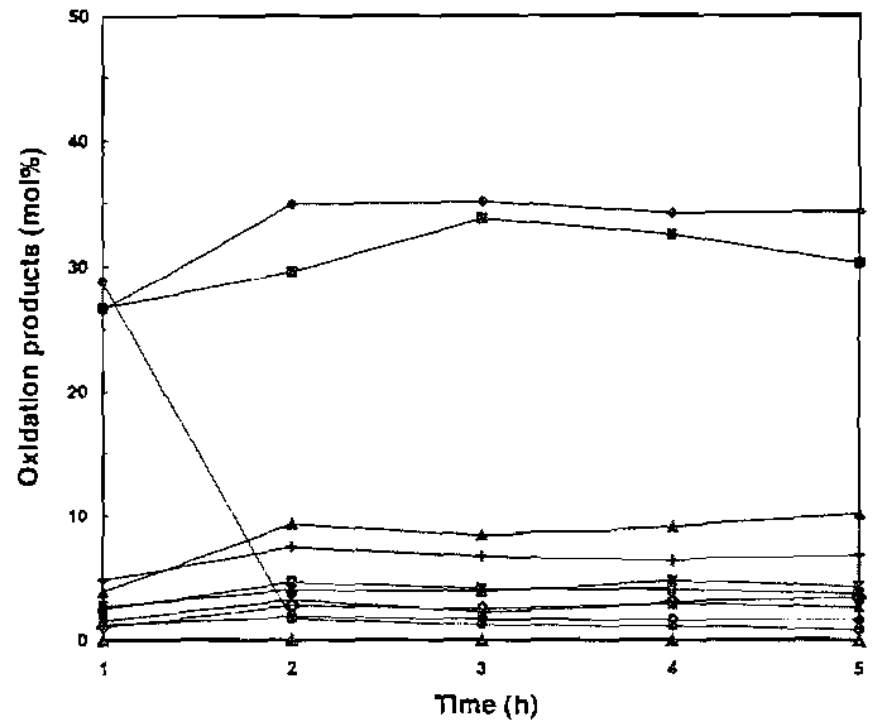

FIG. T. Typical profile of various products formed during the cataiytic oxidation of oleic acid with unsupported tungsic oxide: ( $)$, nonarioic acid; ( $\boldsymbol{D}$ ), azelaic acid; ( $\boldsymbol{A}$ ), octanoic acid; (+), succinic acid, (*), sumeric acid, ( $\square$ ), stearic acid; (S), heptanoic acid; ( $x$ ), hexanoic acid; (O), sebacic acid; ( oleic acid; and ( $\triangle$ ), pimelic acid. concentration was reached in 1 h or less (see Fig. 2). All ocher cases showed a maximum azelaic acid concentration after about 2-3 h (see Figs. 1-6). In all cases studied, nonanoic acid appears to be relatively more stable compared to azelaic acid, which seems to bc degrading faster with prolonged healing in the presence of catalyst.

Transition metal catalysts. In the presence of hydrogen peroxide, transition metals may potentially oxidize to their respecrive oxides. This oxide is believed to be responsible for catalyzing the oxidation of unsaturated fatty acids. From a scale-up perspective, this path could be advantageous if the amount of metal oxidation is low and the consequential rate of formation of the products is comparable with the direct use of oxides. Tungsten, tantalum, molybdenum, zirconium, and niobium in the form of a wire were examined in the initial screening. Molybdenum and tungsten wcre significantly oxidized (total oxidation for molybdenum and $60 \mathrm{wt} \%$ for tungsten) and, although the rate of oxidation of oleic acid was comparable with direct oxidation with tungstic oxide, these catalysts were labeled as economically unfeasible. Zirconium and niobium were very resistant to oxidation with hydrogen peroxide. The reaction products with these two catalysts were similar to the oxidation in the absence of catalyst. Tantalum was the only metal that showed a relatively slow oxidation in the presence of hydrogen peroxide (less than $I w t \%$ ) and the oxidation of oleic acid with tantalum was significant, when compared to the direct use of tantalum oxide. The reaction results for the oxidation of oleic acid with tantalum and tantalum wire are presented in Figure 6.

Conversion. Tungstic oxide supported on silica showed the highest conversion (see Fig. 2). The concentration of oleic acid in the product was about $13 \mathrm{~mol} \%$ afterl h. This corresponds to about $79 \%$ conversion of the initial oleic acid to the

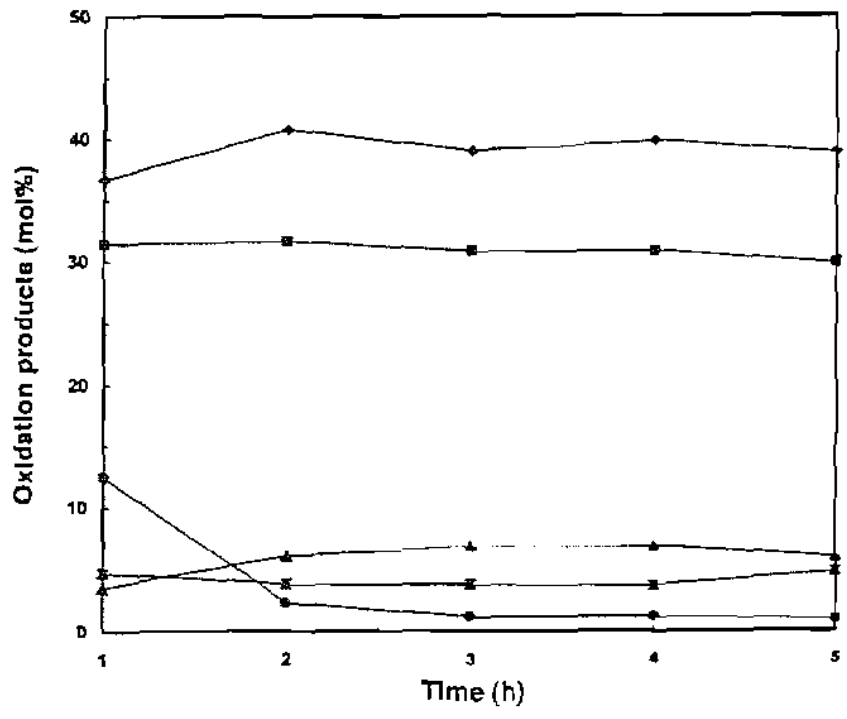

FIG. 2. Reaction results for the oxidation cf oleic acid, subject to silicasupponed tungstic oxide catalyst: ( nonanoic acid; ( $)$ ), azelaic acid;

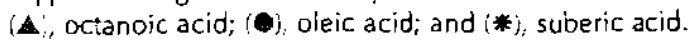




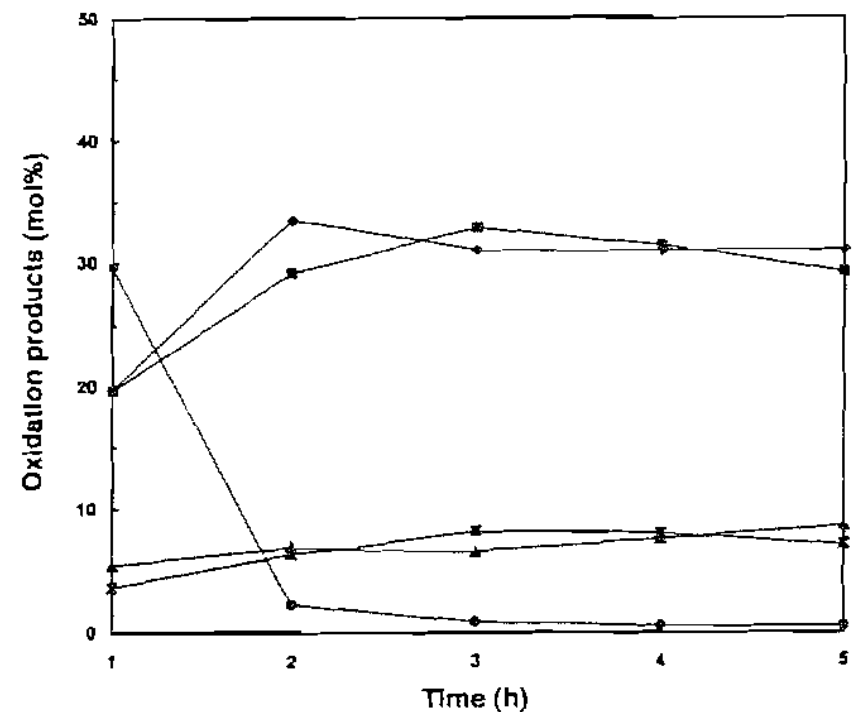

FIG. 3. Reaction resuits for the oxidation of oleic acid, subject to alumina-supported tungstic oxide cataiyst: (\$), nonanoic acid; ( $\mathbf{C})$, azelaic acid; (A), oclanoic acid; (-), oleic acid; and (*), suberic acid.

oxidation products. Conversion of oleic acid was $96 \%$ in $2 \mathrm{~h}$ and about $98 \%$ after $3 \mathrm{~h}$ and longer. When unsupported tungstic oxide was used the concentration of oleic acid in the product was about $29 \%$ and the conversion of oleic acid was at about $56 \%$ after I h (see Fig. 1), which was significantly Iower when compared with supported tungstic oxide. However, the performance of unsupported tungstic oxide was comparaole with the supported catalyst after $2 \mathrm{~h}$ of reaction. The composition of oleic acid was almost the same in both cases after $2 \mathrm{~h}$ of reaction time (see Figs. 1. 2).

Tungstic oxide supported on alumina showed a similar pattern to the silica-supported catalyst as shown in Figure 3. The rate of reaction for alumina-supported catalyst was slightly lower than for silica supported catalyst. The smaller average pore diameter of alumina (135 $\AA$ ) compared to the silica (250 $\AA)$ may have been responsible for the lower rate of reaction for the alumina-supported catalyst. Also alumina pellets tend to settle down in the bottom of the reactor vessel and create diffusion resistances which may result in slower reaction. Using a stirred-contained solids reactor where the catalyst basket is mounted around the paddle or agitator may increase the reactivity of this catalyst. This ensures better contact of reactants with the active sites of the catalyst.

The reaction results for supported and unsupported tantalum oxide are presented in Figures 4 and 5, respectively. When tantalum oxide supported on alumina was used, the performance of the catalyst was relatively poor. Oleic acid concentration in the product was about $18 \mathrm{~mol} \%$, which amounts to about $70 \%$ conversion after about $3 \mathrm{~h}$ of reaction. The maximal conversion that could be attained in $5 \mathrm{~h}$ was about $77 \%$. Moreover, the relative concentrations of nonanoic and azelaic acids with respect to conversion of oleic acid were lower than unsupported tantalum oxide and much lower than supported tungstic oxide, indicative of a high rate of chain

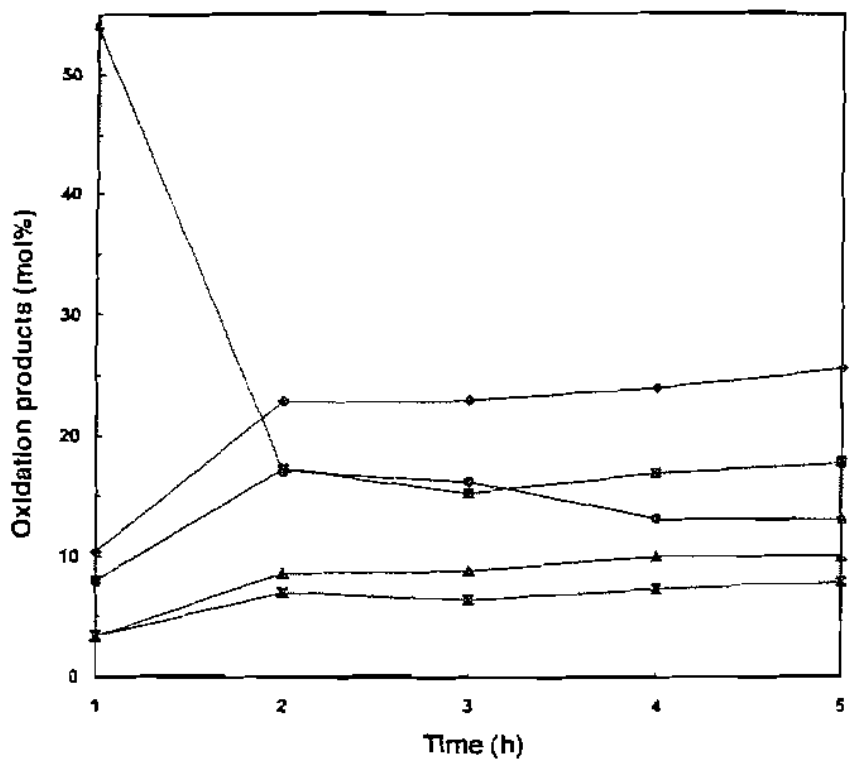

FIG. 4. Reaction results for the oxidation of oleic acid, subject to alumina-5upported tanta!um oxide catalyst: ( nonanoic acid; (m), azelaic acid; (A), octanoic acid; ( ), oieic acid; and (*), suberic acid.

degradation and low selectivity of catalyst for the oxidation of unsarurated bond. The unsupported tantalum oxide resulted in a composition of oleic acid in the product of 11 and 2 mol\% after 1 and 3 h. respectively (see Fig. 5). The corresponding conversion of oleic acid was about 80 and $96 \%$ after 1 and 4 $h$, respectively. This appeared to be higher than conversion with tungstic oxide. However, the concentration of the desired products, i.e., nonanoic and azelaic acids, were much lower for tantalum oxide.

In the case of the oxidation of oleic acid with unsupported metallic tantalum, the concentration of oleic acid in the prod-

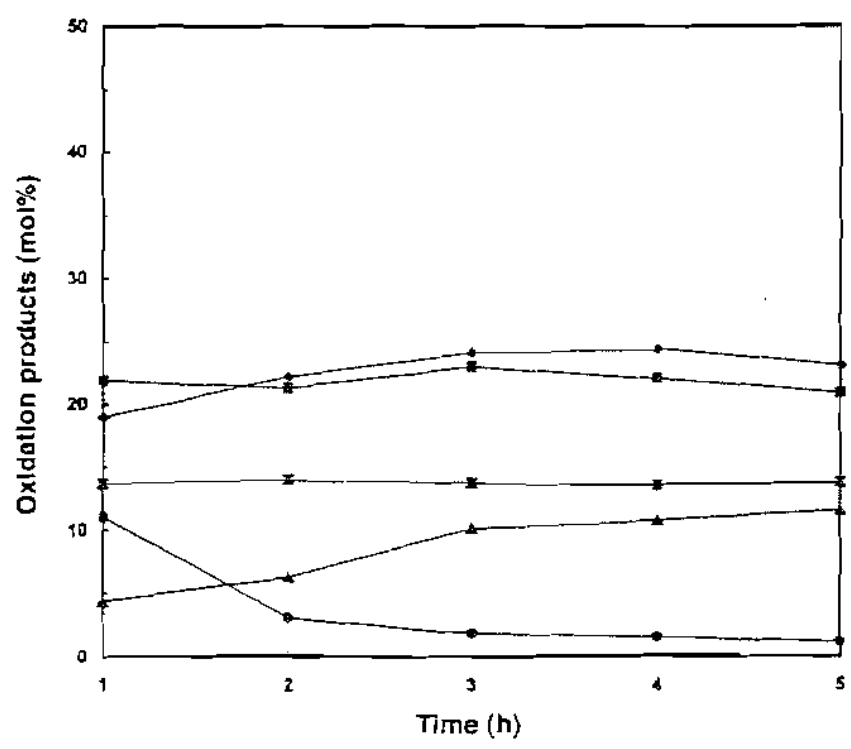

FIG. 5. Reaction results for the oxidation of oleic acid, subject to unsupported tantalum oxide calalyst: ( nonanoic acid; ( $)$, azelaic acid; (A), octanoic acid; ( oleic ac:c; and (*), suberic acid. 
uct was about 36 mole $\%$ after $1 \mathrm{~h}$ and $2 \%$ after $4 \mathrm{~h}$ as shown in Figure 6 . The corresponding conversion of oleic acid was about 52 and $96 \%$ after 1 and 4 h, respectively. Formation of nonanoic and azelaic acids was comparable with tungstic oxide between $2-5$ h of reaction. However. the rate of oxidation for this catalyst was relatively low during the first hour of the reaction. This may have been caused by the slow rate of oxidation of tantalum by hydrogen peroxide to tantalum oxide, which then catalyzes the oxidation of oleic acid.

Selectivity. The selectivity of unsupported tungstic oxide and tungstic oxide supported on silica and alumina were comparable and were marginally different in terms of types and amounts of by-products formed during the seaction (Figs. $1-3)$. Octanoic acid, which was detected as the most concentrated by-product. appeared at a relatively uniform concentration. In all cases. the concentration of pimelic acid was the lowest at about 2 mol\%. Although numerous mono- and dicarboxylic acids were also formed during the course of the reaction, tungstic oxide appeared to be very selective in the oxidation reaction. and for mosc of the systems the concentration of the chain degradation products was under 5 mol\%.

The selectivities of the unsupported tantalum oxide catalysts toward the formation of nonanoic and azelaic acids were less compared to tungstic oxide catalysts (see Figs. 1 and .5). The reactions with the unsupported tanialum oxide caralyst appear to generate a greater quantity of the chain degradation products such as octanoic and suberic acids compared to the unsupported tungstic oxide catalysts. In the case of unsupported tantalum oxide catalyst, the average concentration of octanoic and suberic acids formed was about 11 and 13 mol\%, respectively (Fig. 5), as compared to about 10 and 4 mol\% for the unsupported tungstic oxide (Fig. 1). The relatively high concentration of the lower carbon mono- and

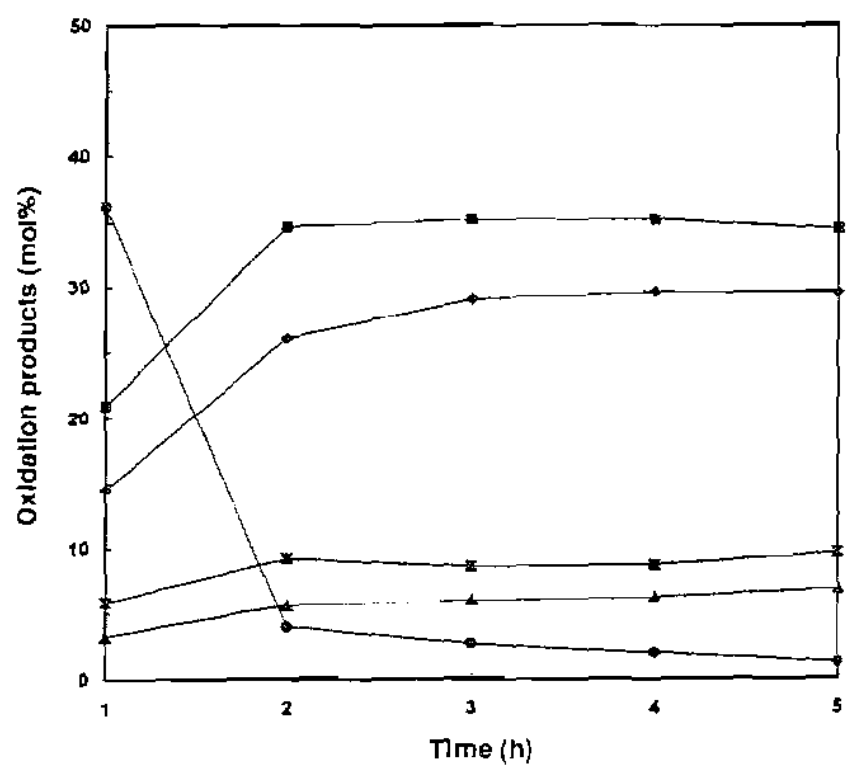

FIC. 6. Reaction results for the oxidation of aleic acid, subject to unsupported metallic tantalum oxide catalyst: (1), nonanoic acid; ( $\mathbf{E})$, azelaic acid; (\$), octanoic acid; i $)$, oleic acid; and (*), suberic acid. diacids early in the reaction media ( 1 h into the reaction) indicative of low selectivity of tantalum oxide for the oxid tion of unsaturation bond of the oleic acid (Fig. 5).

In all cases for tungstic oxide- and tantalum oxide-base catalysts. products were either about equally distributed in th mole percertage of nonanoic and azelaic acids or were mor: concentrated in nonanoic acid than azelaic acid except for un supported tantalum that appeared to bc more selective towar the formation of azelaic acid (see Fig. 6). This may be due to the lower azelaic acid chain degradaijon rate in the presence of transition metal catalysts.

Effect of impurities. In the entire case studies, technical. grade 90 mole percentage oleic acid was used. The main impurity in this acid was determined to be stearic acid. a $\mathrm{C}_{18}$ saturated monocarboxylic acid. Stearic acid appeared to be very stable under the reaction conditions. Its composition remained unchanged throughout the reaction period. To study the effect of impurities during the course of the reaction. oleic acid of $99 \%$ purity was used to establish the baseline for the products. With pure oleic acid, the formation of the products and their quantities were comparable with that of technicalgrade oleic acid. Stearic acid was not detected in the analysis of the reaction products with $99 \%$ oleic acid. Figure 7 shows the profile of products formed from oxidation of pure and technical grade oleic acid with cungstic oxide catalyst supported on silica. This figure reveals that the impurities in technical-grade oleic acid did not interfere with the reaction.

Silica-supported tungsten oxide. One of the objecrives of this study was to develop a feasible process scale-up scheme. A factor of particular interest in the process scale-up is the reaction residence time. It was emphasized earlier that reactions with tungstic oxide supported on silica resulted in the highest conversion after about $1 \mathrm{~h}$. Examination of Figure 2 also reveals that the maximal amount of azelaic acid, the di-acid of interest, was about 32-33 mol\% only after 1 h of reaction. Therefore, it appears that after the first hour of reaction. the rate of oxidation of oleic acid is almost equal to the rate of

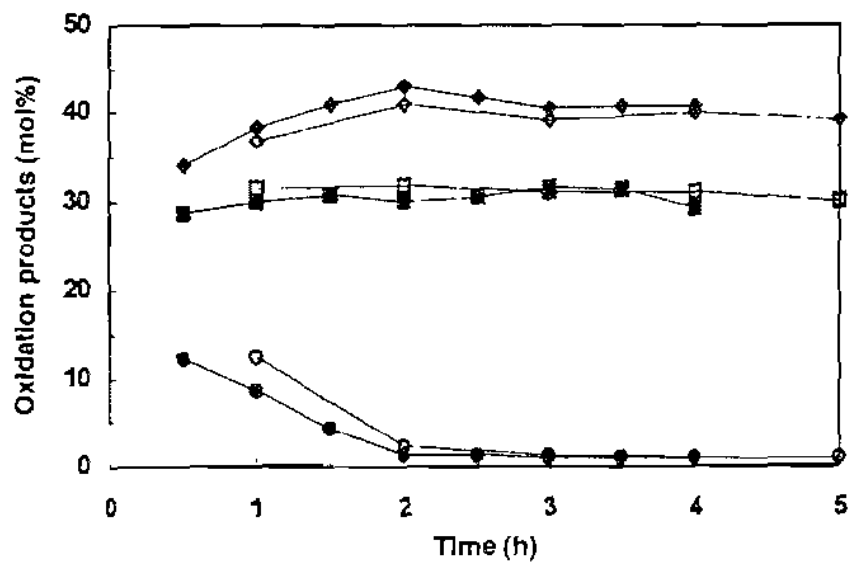

FIG. 7. Reaction results for the oxidation of oleic acid. subject to sil. ica-supported lungstic oxide catalyst: $\diamond_{i}$, nonanoic acid: (ㄴ), azelaic acid; and (O), oleic acid. Solid and open symbols indicate 99 and $90 \%$ o'eic acid, respeciively. 
chain degradation. This explains the somewhat steady concentration of nonanoic and azelaic acids in most cases after the second hour of the reaction (see Figs. 1-7). Therefore. from a scale-up and design perspective, it appears most logi$\mathrm{cal}$ not to continue the reaction longer than $I \mathrm{~h}$.

The oxidation reaction was investigated further for silicasupported tungstic oxide during the first hour of the reaction. The rate of reaction was compared for silica-supported tungstic oxide and unsupported tungstic oxide. The reaction results for the formation of nonanoic and azelaic acids during the first hour of the reaction are presented in Figures 8 and 9 , respectively. Data obtained for the uncatalyzed reaction are also included in these figures. Initially, the unsupported catalyst showed the highest activity. However, while the activity of the unsupported catalyst stayed constant throughout the whole $60 \mathrm{~min}$ of the reaction, the supported catalyst resulted in a significant oxidation after the first $20 \mathrm{~min}$ of the reaction. The low activity during the initial stage of the reaction with the supported catalyst may be due to high pore diffusion resistance that is caused by the support. Once the initial resistance is overcome, the rate of reaction tums out to be much faster than the case of unsupported catalyst. After $1 \mathrm{~h}$ of reaction, the concentration of nonanoic and azelaic acids was 36 and 32 mole percentage, respectively, as compared to 29 and 30 mole percentage in the case of unsupported catalyst. In the case of uncatalyzed reaction, the concentration of nonanoic and azelaic acids was 6 and $7 \mathrm{~mol} \%$, respectively, after $1 \mathrm{~h}$ of the reaction.

\section{ACKNOWLEDGMENTS}

The authors express their gratitude to U.S. Department of Agricul. ture Cooperative State Research Service and Nebraska Soybean Board for their suppor of this work.

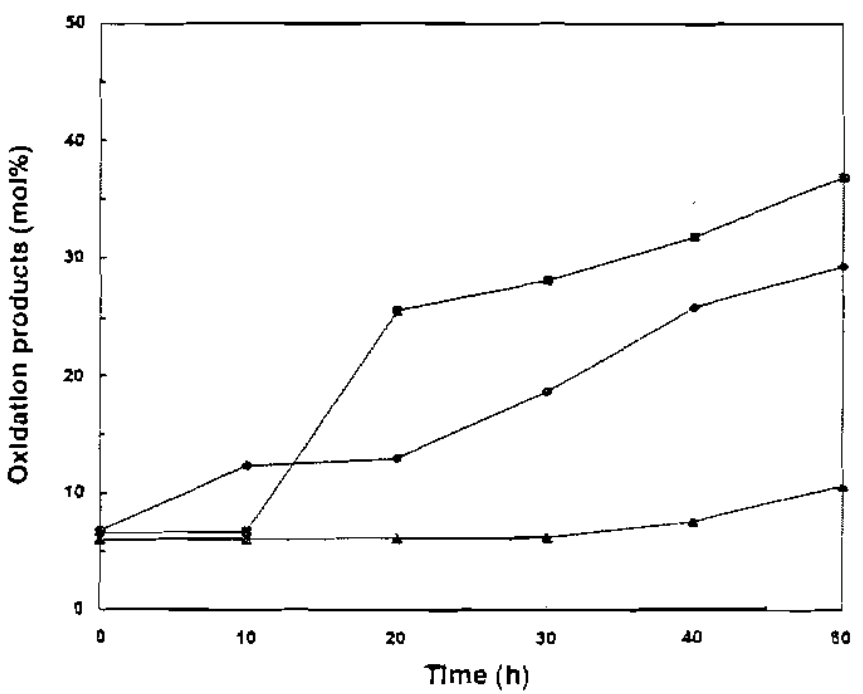

FIG. 8. Formation of nonanoic acid in the oxidation of oleic acid: (1), supported tungstic oxide on silica; (1), unsupported tungstic oxide; and (A), without catalyst.

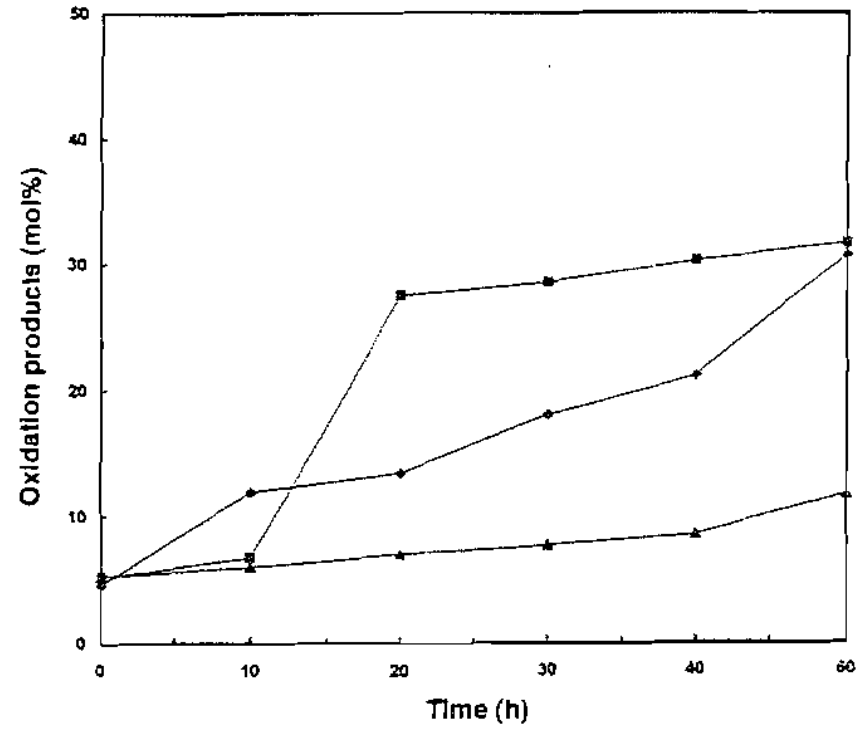

FIG. 9. Formation of azelaic acid in the oxidation of oleic acid: (W), supported tungstic oxide on silica; $(-)$, unsupported tungstic oxide; and (A), without catalyst.

\section{REFERENCES}

1. Johnson, R.W., Dibasic Fatty Acids in Fatty Acids, in Industry, ediced by R.W. Johnson and E. Fritz, Marcel Dekker Inc., New York. 1989, pp. 327-351.

2. Budde, W.W., Jr. General Physical and Chemical Properties of Fatry Acids, in Farry Acids and Their Industrial Applications, edited by S.E. Pattison, Marcel Dekker lnc., New York, 1968, Pp. $47-75$.

3. Kadesch. R.G., Dibasic Acids. J. Am. Oil Chen!. Soc. 3/:568-573 (1954).

4. Kadesch, R.G., Fat-Based Dibasic Acids, Ibid. 56:845A-849A (1979).

5. Nieschlag, H.J., W.H. Tallent. I.A. Wolff, W.E. Palm. and L.P. Witnauer, Ester Plasticizers from Mixed Crambe Dibasic Acids, Ind. Eng. Ciném., Prod. Res. Dev. 6:201-204 (1967).

6. Kenneth, D.C., V.E. Sohns, R.B. Perkins Jr., and E.L. Huffman, Brassylic Acid: Chemical Intermediate trom High-Encic Oils. lbid. 16:95-100 (1977).

7. Nieschlag, H.J., J.A. Rothfus, V.E. Sohns, and R.B. Perkins Ir., Nylon-1313 from Brassylic Acid. Ibid. 16:101-107 (1977).

8. Goebel. C.G., A.C. Brown. H.F. Oehischlaeger. and R.P. Rolfer. Method for Making Aze!aic Acid. U.S. Patent 2.813, I13 (1957).

9. Nieschlag, H.J., I.A. Wolff. T.C. Manley, and R.J. Holland, Brassylic Acid from Ozonolysis of Erucic Acid, Ind. Eng. Prod. Res. Dev. 6:120-123(1967).

10. Gari, N., and E. Avni. Permanganate Oxidation of Oleic Acid Using Emulsion Technoiogy. J. Am. Oil Chem. Soc. 58:840-841 (1981).

11. Zaldman, B., S.Y. Kesliev, and N. Garti. Double Bond Oxidation of Unsaturated Fattv Acids. /bid. 65:611-615 (1988).

12. Hill. J.W.. and W.L. McEwen. Azelaic Acid, Org. Syn., Coll. $2: 5-55(1943)$.

13. Ayorinde, F.O. G. Osman, R.L. Shephard, and F.T. Powers, Synthesis of Azelaic Acid and Suberic Acid from Vernonia galamensis Oil, J. Am. Oil. Chem. Soc. 65:1774-1777 (1988).

14. Passi, S., M. Picardo, C. De Luca. M. Nazzaro-Poro, L. Rossi, and $G$. Rotillo, Saturaied Dicarboxylic Acids as Products of $\mathrm{Un}_{\mathrm{n}}$ saturated Fatty Acid Oxidation, Biochim. Biophys. Acta 1168:190-198 (1993). 
15. Shen, H.C., and H.S. Weng, Liquid-Phase Oxidation of Cyclohexanone to Dibasic Acids with Immobilized Cobalt Catalyst, lnd. Eng. Chem. Res. 27:2254-2260 (1988).

16. Shen, H.C., and H.S. Weng, Liquid-Phase Oxidation of Cyclohexanone over Cerium Oxide Catalyst, lbid. 29:713-719 (1990).

17. Fujitani, K., and M. Nakazawa, A Process for Preparation of Carboxylic Acid. Japanese Patent 240.129 (1986).

18. Nakano, Y., and T.A. Foglia. Oxidation of Linsaturated and Hydroxy Fatty Acids by Ruthenium Tetroxide and Ruthenium Oxyanions. J. Am. Oil Chem. Soc. 59:163-166 (1982).

19. Rushgen, M., P. Klaas, and S. Warwel, Transition-Metal Catalyzed Cleavage of Unsaturated Fatty Acids. Fat Sci. Technol. 97:359-367 (1995).

20. Willms, R.S., D.D. Reible, D.M. Weitzel, and D.P. Harrison. Aqueous-Phase Oxidation: Rate Enhancement Studies, Ind. Eng. Chen. Res. 26:606-612 (1987).

21. Pendyala. K.S.. Catalytic Oxidation of Unsaturated Fatty Acids, M.S. Thesis, University of Nebraska-Lincoln. December 1995.
22. Metcalfe, L.D.. and A.A. Schmitz, The Rapid Preparation of Fatty Acid Esters for Gas Chromarographic Analysis, Anal. Chem. 33:363-364 (1961).

23. Metcalfe, L.D., A.A. Schmitz, and J.R. Pelka, The Rapid Preparation of Fatty Acid Esters for Gas Chromatographic Analysis, Anal. Chem. 38:514-515 (1966).

24. Bannon, C.D., J.D. Craske, N.T. Hai, N.L. Harper, and K.L. ORourke. Analysis of Fatty Acid-Methyl Esters with High Accuracy and Reliability, II. Meihylation of Fars and Oils with Boron Trifuoride-Methanol, J. Chromarogr. 247:63-69 (1982).

25. Chang, I., and W. Sandera, Fast GC Analysis of Free Volarile Organic Acids, Fatty Acids, and Their Isomers Using HP INNOWax Columns and EPC, Hewleti-Packard Company publication, Copyright 1993.

[Received May 26.1998: accepted November 20, 1998] 